\title{
Clinical Update: Literature Abstracts
}

\section{EXISTENTIAL AND SPIRITUAL ISSUES}

\section{The Role of Meaning in Advanced Cancer- Integrating the Constructs of Assumptive World, Sense of Coherence and Meaning-Based Coping}

Lethborg, C., Aranda, S., Bloch, S., and Kissane, D. Journal of Psychosocial Oncology, 24 (2006), 27-42

This study used qualitative methods to elicit the thoughts and attitudes of patients with advanced cancer. Our two interrelated aims were to explore how participants experience and apply meaning and to consider whether this experience can be understood within an integrated framework of assumptive world (AW), sense of coherence (SOC), and meaning-based coping (MBC). Using semistructured interviews, 26 conversations were held overall with 10 participants. Transcriptions were analyzed for themes of lived experience and for evidence of the principal elements of AW, SOC, and MBC. Findings suggest three interrelated domains that form an adaptive pathway toward coherence and sense of self. Although this pathway is essentially linear, it is also responsive to the ongoing stressful nature of advanced cancer.

Religion, Spirituality, and Medicine: Psychiatrists' and Other Physicians' Differing Observations, Interpretations, and clinical approaches

Curlin, F.A., Lawrence, R.E., Odell, S., Chin, M.H., Lantos, J.D., Koenig, H.G., and Meador, K.G.

American Journal of Psychiatry, 164 (2007), 18251831

This study compared the ways in which psychiatrists and nonpsychiatrists interpret the relationship between religion/spirituality and health and address religion/spirituality issues in the clinical encounter. The authors mailed a survey to a stratified random sample of 2000 practicing U.S. physicians, with an oversampling of psychiatrists. The authors asked the physicians about their beliefs and observations regarding the relationship between religion/spirituality and patient health and about the ways in which they address religion/spirituality in the clinical setting. A total of 1144 physicians completed the survey. Psychiatrists generally endorse positive influences of religion/spirituality on health, but they are more likely than other physicians to note that religion/spirituality sometimes causes negative emotions that lead to increased patient suffering ( $82 \%$ vs. $44 \%)$. Compared to other physicians, psychiatrists are more likely to encounter religion/spirituality issues in clinical settings ( $92 \%$ vs. $74 \%$ report their patients sometimes or often mention religion/ spirituality issues), and they are more open to addressing religion/spirituality issues with patients (93\% vs. $53 \%$ say that it is usually or always appropriate to inquire about religion/spirituality). This study suggests that the vast majority of psychiatrists appreciate the importance of religion and/or spirituality, at least at a functional level. Compared to other physicians, psychiatrists also appear to be more comfortable, and have more experience, addressing religion/spirituality concerns in the clinical setting.

\section{Meaninglessness in Terminally Ill Cancer Patients: A Validation Study and Nurse Edu- cation Intervention Trial}

Morita, T., Murata, H., Hirai, K., Tamura, K., Kataoka, J., Ohnishi, H., Akizuki, N., Kurihara, Y., Akechi, T., Uchitomi, Y., and on Behalf of the Japanese Spiritual Care Task Force

Journal of Pain and Symptom Management, 34 (2007), 160-170

Recent empirical studies revealed that fostering patients' perception of meaning in their life is an essential task for palliative care clinicians. However, few studies have reported the effects of training programs for nurses specifically aimed at improving skills to relieve the meaninglessness of terminally ill cancer patients, and we have had no specific measurement instruments. The primary aims of this study were (1) to validate measurement tools 
to quantify nurses' self-reported practice and attitudes toward caring for terminally ill cancer patients feeling meaninglessness and (2) to explore the effects of the 5-hour educational workshop focusing on meaninglessness on nurses' self-reported practice, attitudes toward caring for such patients, confidence, burnout, death anxiety, and meaning of life. A quasiexperimental pre-postquestionnaire survey was performed on 147 nurses. The questionnaire was distributed before the intervention workshop and 1 and 6 months after. The workshop consisted of lecture, role-play, and the exercise of assessment and care planning based on two vignette verbatim records. First, using the first questionnaire sample and an additional sample of 20 nurses for the test-retest examination, we validated a six-item Self-Reported Practice scale and an eight-item Attitudes Toward Caring for Patients Feeling Meaninglessness scale with three subscales (Willingness to Help, Positive Appraisal, and Helplessness). The nurses also completed a scale to assess confidence in caring for terminally ill patients with meaninglessness, the Maslach Burnout Inventory, the Death Attitude Inventory, the Frommelt Attitudes Toward Care of the Dying scale, the Self-Reported Practice Score in General Communication, and the three pain-related items from the Palliative Care Quiz for Nursing. For the Self-Reported Practice scale and the subscales of the Attitudes Toward Caring for Patients Feeling Meaninglessness scale, the Cronbach's alpha coefficients were .63-.91, and the intraclass correlations were .89-.94. The Self-Reported Practice scale significantly, but moderately, correlated with the SelfReported Practice Score in General Communication $(p=.41)$. The Willingness to Help and Helplessness subscales significantly but weakly correlated with the Frommelt scale $(p=-.27, .21)$. Both scales did not correlate or minimally correlated with the Palliative Care Quiz for Nursing $(p<.20)$. The construct validity was confirmed using factor analysis. At the follow-up, of 147 nurses who participated in this workshop, 91 (62\%) and 80 (54\%) nurses responded. Self-reported practice and confidence significantly improved, whereas helplessness, emotional exhaustion, and death anxiety significantly decreased. The percentages of nurses who evaluated this program as "useful" or "very useful" were 79\% (to understand the conceptual framework in caring for terminally ill patients with meaninglessness), 73\% (to help in selfdisclosing nurses' personal beliefs, values, and life goals), and $80 \%$ (to help in learning how to provide care for patients with meaninglessness). The SelfReported Practice scale and the Attitudes Toward Caring for Patients Feeling Meaninglessness scale are reliable and valid tools to specifically quantify nurses' self-reported practice and attitudes toward caring for terminally ill cancer patients feeling meaninglessness of life. The 5-hour workshop appeared to have a modest but significant beneficial effect on nurse-reported practice, attitudes, and confidence in providing care for terminally ill cancer patients feeling meaninglessness. Further educational intervention trials with control groups are promising.

\section{Burden to Others and the Terminally Ill}

Chochinov, H.M., Kristjanson, L., Hack, T., Hassard, T., McClement, S., and Harlos, M.

Journal of Pain and Symptom Management, 34 (2007), 463-471

Studies of patients who are terminally ill consistently identify strong associations between "sense of burden to others" and marked end-of-life distress. However, little research has addressed the issue of burden to others among patients nearing death. The aim of this study was to carefully examine "burden to others" and clarify its relationship with various psychosocial, physical, and existential issues arising in patients who are terminally ill. A cohort of 211 patients with end-stage cancer was assessed, using an assortment of validated psychometrics to document psychosocial, physical, and existential aspects of their end-of-life experience. This included an assessment of their sense of "burden to others." Forty percent of participants indicated a negligible sense of burden to others, scoring within the lowest quarter on an ordinal measure of "burden to others," $25 \%$ scored within the second lowest quarter, $12 \%$ within the third quarter, and $23 \%$ within the highest or most severe range. The most highly correlated variables with "sense of burden to others" included depression $(r=.460, d f=201, p<.0001)$, hopelessness $(r=.420, d f=199, p<.0001)$, and outlook $(r=.362, \quad d f=200, \quad p<.0001)$. Four variables emerged in a multiple regression analysis predicting burden to others, including hopelessness, current quality of life, depression, and level of fatigue $\left(R^{2}\right.$ $\operatorname{adj}=0.32, F[6,174]=13.76, p<.0001)$. There was no association between sense of burden to others and actual degree of physical dependency. Feeling a sense of burden to others is common among dying patients. Although $40 \%$ of the sample reported little in the way of sense of burden to others, the remainder endorsed higher degrees of burden-related distress, with $23 \%$ scoring within the most severe range. The lack of association between "sense of burden to others" and the degree of physical dependency suggests this perception is largely mediated through psychological and existential considerations. Strategies that target meaning and purpose, depression, and 
level of fatigue could lessen this source of distress and enhance quality, dignity-conserving care.

\section{MEASURES}

Factor Structure and Concurrent Validity of the Satisfaction with Life Domains Scale for Cancer (SLDS-C)

Baker, F., Denniston, M., Hann, D., Gesme, D., Reding, D.J., Flynn, T., and Kennedy, J.S.

Journal of Psychosocial Oncology, 25 (2007), 1-17

The Satisfaction with Life Domains Scale for Cancer (SLDS-C) is a brief self-report measure of quality of life that uses a familiar and easily understood graphic response format of smiling and frowning faces. This article explores the factor structure and further examines the validity of the SLDS-C compared with another cancer specific quality-of-life measure, the Functional Assessment of Cancer Therapy Scale-General (FACT-G), in a sample of patients with a variety of cancer diagnoses. A self-administered questionnaire containing the SLDS-C and the FACT-G was completed by 352 adult cancer survivors who were at various points postdiagnosis. Three SLDS-C factor subscales were derived from analyses of these data: "Life as a Whole," "Daily Activities," and "Social Relationships." Evidence for the concurrent validity of the SLDS-C is presented, including its correlation with the total score of the FACT-G $(r=.76)$ and its correlation with subscale scores of this widely used cancer quality-of-life measure. The SLDS-C has a high level of internal consistency $($ alpha $=.94)$, and differences in SLDS-C total scores were found for time since diagnosis, number of diagnoses, spread of disease, type of cancer, and treatment status.

\section{The Social Work Assessment Tool (SWAT)}

Reese, D.J., Raymer, M., Orloff, S.F., Gerbino, S., Valade, R., Dawson, S., Butler, C., Wise-Wright, M., and Huber, R.

Journal of Social Work in End of Life \& Palliative Care, 2 (2006), 65-95.

This paper reports on the last of three National Hospice and Palliative Care Organization initiatives to move hospice and palliative care social workers into the patient/family outcomes arena: the development of the Social Work Assessment Tool. The experience of a team of practitioners and researchers is described, including results of two pilot studies and subsequent SWAT revisions. The major focus is on the current model performance improvement project, in which 19 social workers from 14 hospice and palliative care programs used the SWAT with 101 patients and 81 primary caregivers for a median of 44 days. Quantitative analysis indicated significant improvement in SWAT scores for patients from the first to the second social work visit $(t=-2.60, d f=47$, $p$.01). Qualitative interviewing of the social workers indicated some lack of readiness in the field to conduct quantitative outcomes measurement. Additional measures are needed in addition to the SWAT, including qualitative measures and measures of mezzo and macro practice. Participants indicated that the SWAT was appropriate for use with economically and culturally diverse clients.

\section{The Validation of the Texas Revised Inventory of Grief on an Older Latino Sample}

Wilson, S.

Journal of Social Work in End of Life \& Palliative Care, 2 (2006), 33-60

Bereavement is considered to be one of the most profound experiences in older adulthood. However, assessments of emotional reactions to grief and loss have often been based on measures tested on primarily Anglo samples. This study examined the validity and factor structure of a commonly used bereavement measure on an older Latino sample. Using convergent and discriminant validation procedures, this instrument was tested using a purposive sample of 134 older, recently bereaved Latinos. Although the instrument was originally designed to measure two domains of grief, Past Behaviors and Present Feelings, confirmatory and exploratory factor analysis revealed a three-factor solution for this sample, which included also Disbelief of the Loss. Items within this domain included anger, rejection, and a sense of injustice. This study underscores the need for improved measures in research on grief and bereavement to capture the intensity and severity of grief in a cross-cultural context.

\section{Systematic Review of Observational (Behavioral) Measures of Pain for Children and Adolescents Aged 3 to 18 Years}

von Baeyera, C. and Spagruda, L.

Pain, 127 (2007), 140-150

Observational (behavioral) scales of pain for children aged 3 to 18 years were systematically reviewed to identify those recommended as outcome measures in clinical trials. This review was commissioned by the Pediatric Initiative on Methods, Measurement, 
and Pain Assessment in Clinical Trials (www. immpact.org). In an extensive literature search, 20 observational pain scales were identified for review including behavior checklists, behavior rating scales, and global rating scales. These scales varied in their reliance on time sampling and inclusion of physiological items and facial and postural items as well as their inclusion of multiple dimensions of assessment (e.g., pain and distress). Each measure was evaluated based on its reported psychometric properties and clinical utility. Scales were judged to be indicated for use in specific acute pain contexts rather than for general use. Two scales were recommended for assessing pain intensity associated with medical procedures and other brief painful events. Two scales were recommended for postoperative pain assessment, one for use in hospital and the other at home. Another scale was recommended for use in critical care. Finally, two scales were recommended for assessing pain-related distress or fear. No observational measures were recommended for assessing chronic or recurrent pain, because the overt behavioral signs of chronic pain tend to habituate or dissipate as time passes, making them difficult to observe reliably. In conclusion, no single observational measure is broadly recommended for pain assessment across all contexts. Directions for further research and scale development are offered.

\section{Validation and Psychometric Assessment of a Short Clinical Scale to Measure Chemother- apy-Induced Nausea and Vomiting: The MASCC Antiemesis Tool}

Molassiotis, A., Coventry, P., Stricker, C., Clements, C., Eaby, B., Velders, L., Rittenberg, C., and Gralla, R.

Journal of Pain and Symptom Management, 34 (2007), 148-159

There is a lack of clinical tools to facilitate communication between clinicians and patients about chemotherapy-induced nausea and vomiting (CINV). The Multinational Association of Supportive Care in Cancer (MASCC) has developed such a tool, which is an eight-item scale for the assessment of acute and delayed nausea and vomiting and is completed once per cycle of chemotherapy. The aim of the current study was to assess its psychometric properties, specifically reliability and validity, cultural transferability and equivalence, and congruence with proxy assessments, as well as to determine if accuracy of recall of CINV events using the MASCC Antiemesis Tool (MAT) differed over time from chemotherapy. A prospective study was carried out with adult cancer patients and their informal carers from two hospitals, one each in the United Kingdom and
United States. Patients completed the Rhodes Index for nausea, vomiting, and retching (INVR) daily for the first 5 days after chemotherapy and were then asked to complete the MAT at 1 week, 2 weeks, or 3 weeks after chemotherapy. Carers completed an adapted MAT concurrently with patients. The sample consisted of 87 patients and 22 informal carers. The internal consistency reliability of the scale was high, with Cronbach's alphas of .77 (patient sample) and .82 (carer sample). Responses were similar between the U.K. and U.S. samples in terms of nausea and vomiting, and both samples found the scale easy to use. Contrasted-groups validity (using age as a grouping variable) and concurrent validity (MAT compared with INVR) suggested that the scale is sensitive to detect the different dimensions of CINV and performed well against a daily assessment of nausea/vomiting (total score correlation $r=.86, p<.001$ ). Recall of events was high even 3 weeks after chemotherapy (correlations with INVR of .44-.99, all $p<.01$ ). Factor analysis clearly identified three factors, namely vomiting, acute nausea, and delayed nausea. Proxy assessments by carers were congruent with the patients' responses, especially in relation to vomiting. The MAT is a reliable, valid, clear, and easy-to-use clinical tool that could facilitate discussion between clinicians and patients about their nausea and vomiting experience, thereby potentially aiding treatment decisions. Regular assessment of nausea and vomiting after chemotherapy has the potential to significantly improve CINV management.

\section{SYMPTOM CONTROL}

\section{Fentanyl Buccal Tablet for Relief of Break- through Pain in Opioid-Tolerant Patients with Cancer-Related Chronic Pain}

Slatkin, N.E., Xie, F., Messina, J., and Segal, T.J.

Journal of Supportive Oncology, 5 (2007), 327-334

Fentanyl buccal tablet (FBT) is a new opioid formulation providing rapid-onset analgesia for the treatment of breakthrough pain (BTP). This study evaluated FBT for BTP in opioid-tolerant patients with chronic cancer pain. The study had a randomized, double-blind, placebo-controlled design and was conducted at 30 outpatient treatment centers in the United States. Following open-label titration, patients were randomly assigned to 1 of 18 doubleblind dose sequences ( 7 FBT tablets, 3 placebo) to treat 10 BTP episodes. Pain intensity was measured on an 11-point scale ( $0=$ no pain; $10=$ worst pain). The primary efficacy measure was the sum of pain intensity differences (PIDs) for the first 60 minutes 
(SPID60); secondary efficacy measures included PIDs and pain relief (PR) measured from $5 \mathrm{~min}$ through $2 \mathrm{~h}$. Adverse events (AEs) were recorded. Of 129 patients enrolled, 87 entered the doubleblind phase. SPID60 significantly favored FBT versus placebo (mean $\pm S E, 9.7 \pm 0.63$ vs. $4.9 \pm 0.50$; $p<.0001$ ). Secondary measures also favored FBT: PIDs and PR showed significant differences versus placebo at $10 \mathrm{~min}(0.9$ vs. $0.5 ; 0.815$ vs. 0.606 , respectively, $p<.0001$ ) and all subsequent time points $(p<.0001)$. AEs were typical of opioids (e.g., nausea, dizziness, fatigue). In conclusion, in this study of opioid-tolerant patients with chronic cancer pain and BTP, FBT was efficacious, well tolerated, demonstrated rapid onset of analgesia (within $10 \mathrm{~min}$ ), and had a sustained effect.

Lorazepam, Diphenhydramine, and Haloperidol Transdermal Gel for Rescue from Chemotherapy-Induced Nausea/Vomiting: Results of Two Pilot Trials

Bleicher, J., Bhaskara, A., Huyck, T., Constantino, S., Bardia, A., Loprinzi, C.L., and Silberstein, P.T.

Journal of Supportive Oncology, 6 (2008), 27-32

Despite their use of prophylactic antiemetic therapies, cancer patients continue to consider chemotherapy-induced nausea and vomiting (CINV) to be a significant problem. Patients frequently use various "breakthrough" medications for these symptoms. Unfortunately, there is a paucity of trials regarding treatment of breakthrough CINV. This study investigated the efficacy of "ABH," a topical gel containing lorazepam (Ativan), diphenhydramine (Benadryl), and haloperidol (Haldol), in reducing breakthrough CINV. Adults receiving standard recommended prophylactic antiemetics as outpatients were instructed to use $0.5 \mathrm{ml}$ of the gel topically when they experienced significant CINV. Patients then were contacted retrospectively to respond to a questionnaire rating their nausea and/or vomiting and their response to $\mathrm{ABH}$-gel treatment. The results were collected during two trials: Trial I began in April 2003, and Trial II began in March 2006. During Trial I, 23 patients were evaluated; 17 patients $(74 \%)$ reported that use of the gel decreased their CINV, with 15 (70\%) reporting relief within $30 \mathrm{~min}$ of its application. Three patients believed that the gel caused sedation; no troubles with skin irritation or muscle spasms were reported. In Trial II, all 10 patients believed that the treatment was effective. When the severity of CINV was quantified on a scale of $0-10$, the mean CINV score decreased significantly from a 6.1 before gel application to a 1.7 as evaluated $30 \mathrm{~min}$ following gel application $(p<.005)$. Topical use of ABH gel appears to be a promising and safe rescue therapy for breakthrough CINV that occurs despite prophylactic antiemetic therapy. These results warrant further confirmation in a large, randomized, placebo-controlled trial.

\section{Prescription OxyContin abuse among Patients Entering Addiction Treatment}

Carise, D., Dugosh, K.L., McLellan, A.T., Camilleri, A., Woody, G.E., and Lynch, K.G.

American Journal of Psychiatry, 164 (2007), 17501756

OxyContin and other pharmaceutical opioids have been given special attention in the media, who frequently describe problematic users of the drug as previously drug-naive individuals who become addicted following legitimate prescriptions for medical reasons. The purpose of this study was to characterize the nature and origins of pharmaceutical opioid addiction among patients presenting at substance abuse treatment programs. The authors evaluated the prevalence and correlates of OxyContin use and abuse among a population of 27,816 subjects admitted to 157 addiction treatment programs in the United States from 2001 to 2004. The data collected included the lifetime and past 30-day use of OxyContin and other drugs prior to admission to addiction treatment, source of drug supply, and prior treatment history. Approximately 5\% of all subjects who were admitted to the 157 addiction treatment programs reported prior use of OxyContin. Of those subjects, $4.5 \%$ reported using the drug on a regular basis for at least 1 year, and $2 \%$ reported use of the drug during the 30 days prior to admission. Seventy-eight percent of subjects who reported OxyContin use also reported that the drug had not been prescribed to them for any medical reason, 86\% reported use of the drug to "get high or get a buzz," and 78\% reported receiving prior treatment for a substance use disorder. The patients in this sample did not include individuals from private therapists or pain clinics. However, among treatment-seeking individuals who use OxyContin, the drug is most frequently obtained from nonmedical sources as part of a broader and longer-term pattern of multiple substance abuse.

\section{The Experience of Cancer-Related Fatigue and Chronic Fatigue Syndrome: A Qualitative and Comparative Study}

Bennett, B., Goldstein, D. Friedlander, M., Hickie, I., and Lloyd, A.

Journal of Pain and Symptom Management, 34 (2007), 126-135 
Cancer-related fatigue (CRF) is a common and disabling symptom complex reported by survivors. This study aimed to better understand the manifestations of CRF in women treated for breast cancer, and to compare them with those of women diagnosed with chronic fatigue syndrome (CFS). Women with CRF persisting 6 months after treatment for early stage breast cancer and women with CFS participated in separate, audiotaped focus groups. Transcripts of the sessions were analyzed using the NUD*IST software and interpreted using grounded theory. Twenty-eight women participated, 16 with CRF and 12 with CFS. Analysis of transcripts from both groups revealed a similar core set of symptoms, featuring fatigue, neurocognitive difficulties, and mood disturbances. Women with CFS reported additional symptoms including musculoskeletal pain and influenza-like manifestations. Both groups suffered disabling behavioral consequences of the symptom complex. Qualitatively, $\mathrm{CRF}$ appears closely related to CFS. These findings raise the emergent hypothesis of a conserved neurobehavioral symptom complex, which results from diverse triggering insults.

\section{Preference for Analgesic Treatment for Cancer Pain among African Americans}

Meghani, S. and Keane, A.

Journal of Pain and Symptom Management, 34 (2007), 136-147

Over the past decade, there has been a surge of literature related to disparities in pain treatment between minority and nonminority patients. Conspicuously lacking from this body of literature is an effort to investigate the patient-level factors that might account for undertreatment of pain among minorities. The present qualitative descriptive study was designed to gain a preliminary understanding of the preference for analgesic treatment for cancer pain among African Americans and the factors shaping these preferences. The sample $(n=35)$ was recruited from three outpatient oncology clinics in the Mid-Atlantic region. Inclusion was based on self-identified African Americans, $>18$ years of age, diagnosed with solid tumors, with self-reported cancer pain of at least 1 month, and no history of major surgery in the past 3 months. The data were gathered using demographics, the Brief Pain Inventory-Long Form, and in-depth semistructured interviews. Qualitative findings revealed that despite having overt access to pain medications, considerable intra-ethnic heterogeneity existed in the preference for analgesia among this group of African Americans. The subjective preference for analgesics for cancer pain was tied to a number of covert factors such as meaning of cancer pain treatment, past experience with pain relief and analgesic side effects, fears of dependency and tolerance, and past experience with providers and the health system. These factors should be the focus of future inquiry.

\section{Gabapentin and an Opioid Combination versus Opioid Alone for the Management of Neuro- pathic Cancer Pain: A Randomized Open Trial}

Keskinbora, K., Ferit Pekel, A., and Aydinli, I.

Journal of Pain and Symptom Management, 34 (2007), 183-189

Neuropathic cancer pain represents a major challenge. Treatment often requires adjuvant analgesics, including gabapentin, to complement the effects of opioids. This study aimed to compare the effectiveness and safety of gabapentin combined with an opioid versus opioid monotherapy for the management of neuropathic cancer pain. Seventy-five cancer patients who were receiving opioid therapy and reported sufficient pain relief of nociceptive, but not neuropathic, pain were enrolled. Sixty-three patients completed the study. Patients were randomized to one of the following treatment protocols: (1) gabapentin adjuvant to ongoing opioid treatment titrated according to pain response while opioid dose was kept constant (group $\mathrm{GO}$ ), and (2) continuation of opioid monotherapy according to the World Health Organization treatment ladder approach (group OO). Changes in pain intensity, allodynia, and analgesic drug consumption were evaluated at Day 4 and Day 13. Side effects were also recorded. Both treatments resulted in a significant reduction of pain intensity at Day 4 and Day 13 compared to baseline. However, mean pain intensity for burning and shooting pain was significantly higher in the OO group compared to the GO group at both the 4 th $(p=.0001)$ and 13 th $(p=.0001)$ days of the study. An earlier significant decrease (at Day $4, p=.002$ ) was observed for allodynia in the GO group compared to the OO group. The rate of side effects in the GO group was significantly lower than that in the $\mathrm{OO}$ group ( $p=.015$ ). These data suggest that gabapentin added to an opioid provides better relief of neuropathic pain in cancer patients than opioid monotherapy; this combination of gabapentin and an opioid may represent a potential first-line regimen for the management of pain in these patients.

Time Course and Characteristics of Symptom Distress and Quality of Life at the End of Life

Kutner, J., Bryant, L., Beaty, B., and Fairclough, D.

Journal of Pain and Symptom Management, 34 (2007), 27-236 
This study sought to describe the characteristics and correlates of symptom distress and quality of life (QOL) among persons receiving hospice/palliative care. English-speaking adults $(n=86)$, their nurses $(n=86)$, and family caregivers $(n=49)$ from 11 hospice/palliative care organizations completed the Memorial Symptom Assessment Scale (MSAS) and McGill Quality of Life Questionnaire (MQOL) at hospice/palliative care enrollment, at 1 week and 2 weeks, then monthly until death or discharge. Mixed effects modeling using proxy reports to impute missing patient-reported data was used to describe predictors of symptom distress and QOL. Given study population attrition due to death, analyses are limited to the first 17 days following hospice/palliative care admission. Although lack of energy and pain were the most prevalent and distressing symptoms (prevalence $92 \%$ and $82 \%$, respectively; mean MSAS scores 3.27 and 2.71, respectively), pain was identified as the most distressing symptom based on its contribution to MSAS summary scores and responses to a single-item "most distressing symptom" question. Pain, nonpain symptom distress, and MQOL scores remained fairly stable throughout the study period. Distress from all other physical symptoms was significantly associated with distress due to pain. There were no significant associations between patient characteristics and distress due to pain. Although greater psychological symptom distress had a negative association with QOL, neither pain nor other physical symptom distress was associated with QOL. The persistence of significant symptom distress, particularly due to pain, argues for the need for enhanced evidence to guide care provided in the last days and weeks of life.

\section{Symptom Management for Cancer Patients: A Trial Comparing Two Multimodal Interven- tions}

Sikorskii, A., Given, C., Given, B., Jeon, S., Decker, V., Decker, D., Champion, V., and McCorkle, R.

Journal of Pain and Symptom Management, 34 (2007), 253-264

The results of a randomized controlled trial that tested the effects of 8-week, six-contact multidimensional interactive interventions for symptom management are presented. Four hundred thirty-five cancer patients with solid tumors undergoing chemotherapy were randomized to receive either nurse-assisted symptom management (NASM) or automated telephone symptom management (ATSM). A prior trial established the effectiveness of NASM compared with conventional care. Seventeen symptoms commonly experienced by patients undergoing chemotherapy were rated on a scale from 0 to 10 and were evaluated at baseline, at each of the six intervention contacts, and postintervention observation at 10 weeks. Both groups achieved significant reduction in symptom severity over baseline, and there was no difference between groups on symptom severity at 10 weeks. Randomization accounted for possible reductions in severity due to response shifts. Severity of symptoms reported by patients at each of the six intervention contacts was measured using a Rasch model. Symptom pattern was different for lung and non-lung cancer patients, and they were analyzed separately. Longitudinal analyses revealed that lung cancer patients with greater symptom severity withdrew from later intervention contacts of the ATSM. The results suggest that both NASM and ATSM achieved a clinically significant reduction in symptom severity. The NASM may be more effective than ATSM in retaining lung cancer patients in the intervention. Further testing of ATSM supplemented by NASM for patients with severe symptoms is warranted.

\section{The Effects of Family Support, Anxiety, and Post-Treatment Nausea on the Development of Anticipatory Nausea: A Latent Growth Model}

Kim, Y. and Morrow, G.

Journal of Pain and Symptom Management, 34 (2007), 265-276

Although the degree of a patient's anxiety and symptoms of posttreatment nausea have been suggested as predictors of anticipatory nausea, little attention has been given to the impact of family support on the development of anticipatory nausea. This study examines the role of family support in the development of the severity of anticipatory nausea, both directly and mediated through a patient's anxiety. Five hundred thirty-nine patients with breast cancer were studied. The results from latent growth modeling showed that family support was associated with the severity of anticipatory nausea mediated by the levels of a patient's anxiety and posttreatment nausea severity. In addition, family support had a direct impact on the severity level of anticipatory nausea. The findings suggest that helping patients and their families communicate in more satisfactory and supportive ways and maintain an organized family system might be beneficial in reducing the symptoms of chemotherapy-related nausea. 
Pain in the Nursing Home: Assessment and Treatment on Different Types of Care Wards

Achterberg, W., Pot, A.M., Scherder, E., and Ribbe, M.

Journal of Pain and Symptom Management, 34 (2007), 480-487

The assessment and management of pain in nursing homes have been shown to be suboptimal, but no study has evaluated differences in clinical setting within these homes. The prevalence and management of pain on different care wards (psychogeriatric, somatic, and rehabilitation) were studied on 562 newly admitted Dutch nursing home residents. Pain was measured according to the Nottingham Health Profile (perceived pain) and the Minimum Data Set pain observation items (frequency and intensity). Pain frequency differed significantly across the different ward types: on psychogeriatric wards $(n=247)$, it was $27.1 \%$; on somatic wards $(n=181), 53.9 \%$; and on rehabilitation wards $(n=129), 57.8 \%$. Being admitted on a psychogeriatric ward was significantly related to less pain compared to being admitted on a somatic ward, even when adjusted for possible confounders such as age, gender, cognitive status, activities of daily living, pain-related disorders, and depression (odds ratio [OR] 0.38 [95\% confidence interval $(\mathrm{CI})=0.23-0.62]$ ). Patients on psychogeriatric wards who had pain received less pain medication, adjusted for frequency and intensity of pain (OR $0.37[95 \% \mathrm{CI}=0.23-0.59]$ ), compared to patients on somatic wards. We conclude that admission to a psychogeriatric care ward, independent of cognition, is associated with lower pain prevalence and also with lower levels of pain treatment.

\section{QUALITY OF PALLIATIVE CARE}

\section{Patients' and Doctors' Views of Using the Sche- dule for Individual Quality of Life in Clinical Practice}

Kettis-Lindblad, A., Ring, L., Widmark, E., Bendtsen, P., and Glimelius, B.

\section{Journal of Support Oncology, 5 (2007), 281-287}

This study explored patients' and oncologists' perceptions of using a computer-administered, individualized quality-of-life (QOL) instrument to support an oncologic consultation. Twenty patients with gastrointestinal cancer ( $50 \%$ female; mean age 60 years) at two hospitals in Sweden completed the Schedule for the Evaluation of Individual Quality of LifeDirect Weight (SEIQoL-DW) and the DiseaseRelated (DR) SEIQoL-DW and brought the results to the consultation. Afterward, interviews were conducted with all patients and six of eight doctors. All interviews were audiotaped, transcribed, and analyzed using an interpretivist approach. Most patients and doctors believed that the instrument would facilitate detection of patients' areas of concern and would support monitoring of patients' QOL. This tool may empower the patient, give the doctor a broader picture of the patient, and influence clinical decision making. The SEIQoL-DW may be a systematic method of stimulating patient-centered care. It apparently encourages patients to reflect upon their own situation and allows them to be seen as whole persons. However, further quantitative evaluation of the intervention's outcomes is required.

\section{Screening for Psychosocial Distress: A Na- tional Survey of Oncologists}

Pirl, W.F., Muriel, A., Hwang, V., Kornblith, A., Greer, J., Donelan, K., Greenberg, D.B., Temel, J., Schapira, L.

\section{Journal for Supportive Oncology, 5 (2007), 499-504}

Little is known about the dissemination and uptake of National Comprehensive Cancer Network (NCCN) guidelines for psychosocial distress in oncology practice. This study surveyed oncologists about their awareness of NCCN guidelines on psychosocial distress and their methods of screening patients for distress. In all, 1000 oncologists practicing in the United States who were members of the American Society of Clinical Oncology were asked to complete an anonymous questionnaire. Predictors of routine screening for distress were identified using logistic regression. Overall, 46\% (448/965) of the oncologists responded. Almost two thirds $(63.4 \%)$ practiced in the community, $27.2 \%$ practiced in cancer centers, and $6.9 \%$ practiced in hospitals. Less than one third (32.3\%) reported being at least somewhat familiar with NCCN guidelines. Two thirds $65.0 \%$; $95 \%$ confidence interval, 60.6-69.4) reported screening patients for distress routinely, but only $14.3 \%$ used a screening instrument. Independent predictors for screening patients for distress included availability of mental health services, knowledge of NCCN guidelines, experience, lack of time, uncertainty about identifying distress, and female gender of the practitioner. NCCN guidelines for psychosocial distress do not appear to be widely disseminated. Whereas the majority of oncologists reported routinely screening patients for distress, only a small percentage followed the guidelines by using a screening instrument. Future efforts should focus on the dissemination and validation of the NCCN guidelines. 
Survival Prediction in Terminally Ill Cancer Patients by Clinical Estimates, Laboratory Tests, and Self-Rated Anxiety and Depression

Gripp, S., Moeller, S., Bölke, E., Schmitt, G., Matuschek, C., Asgari, S., Asgharzadeh, F., Roth, S., Budach, W., Franz, M., and Willers, R.

Journal of Clinical Oncology, 25 (2007), 3313-3320

Our aim was to study how survival of palliative cancer patients relates to subjective prediction of survival, objective prognostic factors (PFs), and individual psychological coping. Survival was estimated according to three categories ( $<1$ month, 1 to 6 months, and $>6$ months) by two physicians (A and B) and the institutional tumor board (C) for 216 patients recently referred for palliative radiotherapy. After 6 months, the accuracy of these estimates was assessed. The prognostic relevance of clinical symptoms, performance status, laboratory tests, and selfreported emotional distress (Hospital Anxiety and Depression Scale) was investigated. In $61 \%$, 55\%, and $63 \%$ of the patients, prognoses were correctly estimated by A, B, and C, respectively. Kappa statistic showed fair agreement of the estimates, which proved to be overly optimistic. Accuracy of the three estimates did not improve with increasing professional experience. In particular, the survival of $96 \%, 71 \%$, and $87 \%$ of patients who died in less than 1 month was overestimated by $\mathrm{A}, \mathrm{B}$, and $\mathrm{C}$, respectively. On univariate analysis, 11 of 27 parameters significantly affected survival, namely performance status, primary cancer, fatigue, dyspnea, use of strong analgesics, brain metastases, leukocytosis, lactate dehydrogenase (LDH), depression, and anxiety. On multivariate analysis, colorectal and breast cancer had a favorable prognosis, whereas brain metastases, Karnofsky performance status less than $50 \%$, strong analgesics, dyspnea, LDH, and leukocytosis were associated with a poor prognosis. This study revealed that physicians' survival estimates were unreliable, especially in the case of patients near death. Self-reported emotional distress and objective PFs may improve the accuracy of survival estimates.

Timing of Referral to Hospice and Quality of Care: Length of Stay and Bereaved Family Members' Perceptions of the Timing of Hospice Referral

Teno, J., Shu, J., Casarett, D., Spence, C., Rhodes, R., and Connor, $\mathrm{S}$.

Journal of Pain and Symptom Management, 34 (2007), 120-125
Previous research has noted that many persons are referred to hospice in the last days of life. The National Hospice and Palliative Care Organization collaborated with Brown Medical School to create the Family Evaluation of Hospice Care (FEHC) data repository. In 2005, 106,514 surveys from 631 hospices were submitted with complete data on the hospice length of stay and bereaved family member perceptions of the timing of hospice care. Of these surveys, $11.4 \%$ of family members believed that they were referred "too late" to hospice. This varied from $0 \%$ to $28.1 \%$ among the participating hospice programs with 30 or more surveys. Among those with hospice lengths of stay of less than a month, only $16.2 \%$ reported they were referred "too late." Although the bereaved family member perceptions of the quality of end-of-life care did not vary by length of stay for each of the FEHC domains, the perception of being referred "too late" was associated with more unmet needs, higher reported concerns, and lower satisfaction. Our results suggest that family members' perception of the timing of hospice referral-not the length of stay-is associated with the quality of hospice care. This perception varies substantially among the participating hospice programs. Future research is needed to understand this variation and how hospice programs are delivering high quality of care despite short length of stay.

\section{Forgoing Artificial Nutrition or Hydration in Patients Nearing Death in Six European Countries}

Buiting, H., van Delden, J., Rietjens, J., OnwuteakaPhilipsen, B., Bilsen, J., Fischer, S., Löfmark, R., Miccinesi, G., Norup, M., and van der Heide, A.

Journal of Pain and Symptom Management, 34 (2007), 305-314

Whether or not artificial nutrition or hydration (ANH) may be forgone in terminally ill patients has been the subject of medical and ethical discussions. Information about the frequency and background characteristics of making decisions to forgo ANH is generally limited to specific clinical settings. The aim of this study was to compare the practice of forgoing ANH in six European countries: Belgium, Denmark, Italy, The Netherlands, Sweden, and Switzerland. In each country, random samples were drawn from death registries. Subsequently, the reporting physician received a questionnaire about the medical decisions that preceded the patient's death. The total number of deaths studied was 20,480 . The percentage of all deaths that were preceded by a decision to forgo ANH varied from $2.6 \%$ in 
Italy to $10.9 \%$ in The Netherlands. In most countries, decisions to forgo ANH were more frequently made for female patients, patients aged 80 years or older, and for patients who died of a malignancy or disease of the nervous system (including dementia). Of patients in whom ANH was forgone, 67\%-93\% were incompetent. Patients in whom ANH was forgone did not receive more potentially life-shortening drugs to relieve symptoms than other patients for whom other end-of-life decisions had been made. Decisions to forgo ANH are made in a substantial percentage of terminally ill patients. Providing all patients who are in the terminal stage of a lethal disease with ANH does not seem to be a widely accepted standard among physicians in Western Europe.

\section{What Predicts the Quality of Advanced Cancer Care in Latin America? A Look at Five Countries: Argentina, Brazil, Cuba, Mexico, and Peru}

Torres Vigil, I., Aday, L., De Lima, L., and Cleeland, C.

Journal of Pain and Symptom Management, 34 (2007), 315-327

Cancer is now a leading cause of death among adults in most Latin American nations. Yet, until recently, there has been limited research on the quality of, and access to, advanced cancer care in developing regions such as Latin America. This landmark, cross-national study assessed the quality of advanced cancer care in five Latin American countries by surveying a convenience sample of 777 physicians and nurses, and identifying the most salient influences on their quality-of-care assessments based on multiple linear regression analyses. Strategies for disseminating this survey included mass mailings, distribution at professional meetings/conferences, collaboration with Latin American institutions, professional organizations, and the Pan American Health Organization, and online posting. Results indicate that the respondents' assessments of the quality of, access to, and affordability of advanced cancer care varied significantly across nations $(p<.001)$. The strongest predictor of providers' national-level assessments of the quality of care was their ratings of access to advanced cancer care (Beta $=.647)$. Other predictors included affordability of care, country (Cuba vs. the other four countries), income-gap quintile, and institutional availability of opioid analgesics. Low prioritization of palliative care in both health care policy formulation and provider education also predicted the quality-of-care ratings. Findings from this study suggest that providers from five different nations hold similar equitable notions of quality care that are dependent on the provision of accessible and affordable care. Measures of social equity, such as the income-gap quintile of nations, and measures of policy barriers, such as the scale developed in this study, should be replicated in future studies to enable policy makers to assess and improve advanced cancer care in their countries.

\section{African American Bereaved Family Members' Perceptions of the Quality of Hospice Care: Lessened Disparities, but Opportunities to Improve Remain}

Rhodes, R., Teno, J., and Connor, S.

Journal of Pain and Symptom Management, 34 (2007), 472-479

Previous research has documented striking disparities in bereaved family members' perceptions of the quality of end-of-life care between African American and white decedents. Using data from the 2005 repository of the Family Evaluation of Hospice Care survey, we examined whether this disparity in quality of end-of-life care persists once an African American is enrolled in hospice. Of the 121,817 decedents whose proxies were surveyed, 4095 were non-Hispanic black (African American), and 97,525 were non-Hispanic white. There were no statistically significant differences with regard to decedents' gender. Length of stay on hospice was similar across racial groups. Although previous research has demonstrated striking disparities in the perceived quality of end-of-life care, we found that there were either no differences (quality ratings scores) or less of a disparity in perceptions of concerns with the quality of end-of-life care when compared to the results of a previously reported national mortality follow-back survey, suggesting that though disparities in perceptions of care at end of life persist, on hospice they improve to some degree.

\section{Does Dying at Home Influence the Good Death of Terminal Cancer Patients?}

Yao, C.-A., Hu, W.-Y., Lai, Y.-F., Cheng, S.-Y., Chen, C.-Y., and Chiu, T.-Y.

Journal of Pain and Symptom Management, 34 (2007), 497-504

To investigate whether dying at home influences the likelihood that a terminal cancer patient will achieve a good death despite the limited medical resources available in many communities, this study investigated the relationship between the achievement of a good death and the performance of good-death services in two groups with different places of death and explored the possible factors associated with this 
relationship. Three hundred seventy-four consecutive patients with terminal cancers admitted to a palliative care unit were enrolled. Two instruments, the good-death scale and the audit scale for gooddeath services, were used in the study. Mean age of the 374 patients was $65.45 \pm 14.77$ years. The total good-death score in the home-death group $(n=307)$ was significantly higher than that in the hospitaldeath group $(n=67)$, both at the time of admission $(t=-5.741, p<.001)$ and prior to death $(t=-3.027$, $p<.01)$. However, the score of item "degree of physical comfort" assessed prior to death in the home-death group was lower than that in the hospital-death group $(p=.185)$. As to the audit scale for good-death services, each subscale score and total scores in the home-death group were significantly higher than that in the hospital-death group, with the exception of the subscale "continuity of social support" (4.72 vs. $4.61, p=.132$ ). Bereavement support $($ odds ratio $=1.01,95 \%$ confidence interval $=$ $0.62-1.39$; multiple regression), alleviation of anxiety $(0.81,0.46-1.15)$, decision-making participation $(0.61,0.26-0.95)$, fulfillment of last wish $(0.45,0.08-0.82)$, and survival time $(0.00,0.00-$ 0.01 ) were independent correlates of the good-death score (35.8\% of explained variance). However, the place of death was not in the model. The study conclusively suggests the necessity for palliative home care to strengthen the competence of physical care. Moreover, earlier incorporation of palliative care into anticancer therapies can lead to better death preparation and good-death services and thus be helpful to achieve a good death.

\section{COMMUNICATION}

\section{Communicating about Chemotherapy-Induced Anemia}

Davidson, B., Blum, D., Cella, D., Hamilton, H., Nail, L., and Waltzman, R.

Journal of Supportive Oncology, 5 (2007), 36-40, 46

Many validated instruments exist for determining the impact of chemotherapy-induced anemia and related fatigue on patient quality of life, but few studies analyze how health care providers actually discuss these subjects with patients. The authors share their study results on patterns of communication between participating patients and their physicians and allied health professionals. Letters of invitation were mailed to over 1000 community-based oncologists, 15 of whom met the criteria and agreed to participate in this study on a first-enrolled basis until sufficient participation was ensured. In total, 36 of their patients were audio- and/or video-recorded during their regularly scheduled visits. Postvisit interviews were conducted separately with patients and participating health care professionals. Interviews were transcribed and analyzed using sociolinguistic techniques. Although $52 \%$ of visit time was spent discussing side effects and symptoms, most discussions of anemia and fatigue lacked specificity necessary to determine their true impact on patients' lives. Physician inquiries regarding fatigue also tended to be too brief to elicit patients' chief concerns. Vocabulary used to discuss anemia and related fatigue was variable and imprecise, and no fatigue assessment instrument was used or referenced in any visit. Community-based oncologists are encouraged to modify their vocabulary and consider incorporating a validated fatigue instrument, either within or before the consultation, to improve the quality of such communication.

\section{PSYCHOSOCIAL INTERVENTIONS}

\section{Relation of Lifetime Trauma and Depressive Symptoms to Mortality in HIV}

Leserman, J., Pence, B.W., Whetten, K., Mugavero, M.J., Thielman, N.M., Swartz, M.S., and Stangl, D.

American Journal of Psychiatry, 164 (2007), 17071713

In an era of highly active antiretroviral therapies, the authors needed to confirm previous findings showing that stress and depression have an impact on HIV disease progression. The goal of the current study was to examine the effects of lifetime trauma, recent stressful events, and depression on all-cause and AIDS-related mortality among HIV-infected men and women. The authors hypothesized that these psychosocial variables would predict significantly faster HIV-specific and all-cause mortality. The authors consecutively sampled HIV-infected men and women who received care at one of eight infectious diseases clinics in five Southeastern states. The sample included 490 patients who were followed by interview for 27 months and followed with their medical records for up to 41 months. There were 29 deaths; 16 were AIDS related. More lifetime trauma and antigenic marker on helper/inducer $\mathrm{T}$ cells (CD4) $<200$ significantly predicted faster all-cause and AIDS-related mortality. For those at or above the median in trauma, the all-cause death rate was 3.54 per 100 person-years, compared to 1.72 for those below the median. For those at or above the median in trauma, the AIDS-related death rate was 2.13 per 100 person-years, compared to 0.77 for those 
below the median. Depressive symptoms and higher baseline viral load were significantly related to greater risk of AIDS-related mortality. Further research is needed to determine if interventions to address trauma and depression can modify these detrimental effects on HIV.

\section{Patient Preferences Regarding Cancer Group Psychotherapy Interventions: A View from the Inside}

Sherman, A.C., Pennington, J., Latif, U., Farley, H., Arent, L., and Simonton, S.

Psychosomatics, 48 (2007), 426-432

Group interventions for cancer patients have commanded notable interest among investigators, but utilization rates are low and little is known about the features that patients themselves deem most important. The authors examined the views of potential participants, among 425 patients with diverse malignancies. A large number (64.6\%) expressed interest, although few had attended a group. Preferences were strongest for interventions convened during diagnostic or active treatment periods rather than later and those focusing on medical education or healthpromotion, rather than emotional support or coping. Most were amenable to drop-in formats and to heterogeneous membership. In subgroup analyses, preferences were associated with disease site and not strongly related to psychosocial or demographic predictors. Understanding patient preferences may be critical for successful program development and utilization.

\section{Parental Grief after Losing a Child to Cancer: Impact of Professional and Social Support on Long-Term Outcomes}

Kreicbergs, U.C., Lannen, P., Onelov, E., and Wolfe, J.

Journal of Clinical Oncology, 25 (2007), 3307-12

It is still uncertain whether or not parents can ever come to terms with the loss of a child and whether professional or social support facilitate the longterm grief process. A Swedish population-based study, which sent an anonymous, mail-in questionnaire to parents who had lost a child to a malignancy 4 to 9 years earlier, gained the participation of 449 $(80 \%)$ of 561 parents. Parents were asked whether, and to what extent, they had worked through their grief. Questions were also asked regarding those who provided parents with support. We examined candidate factors to determine their associations with greater likelihood of working through parental grief. Overall, most parents (74\%) stated that they had worked through their grief "a lot" or "completely" at the time of the follow-up. Parents who had shared their problems with others during the child's illness (fathers: relative risk [RR], 3.0; 95\% CI, 1.8 to 5.0; mothers: RR 1.9; 95\% CI, 1.2 to 2.8 ) and who had access to psychological support during the last month of their child's life (fathers: RR 1.4; 95\% CI, 1.0 to 1.8; mothers: RR 1.3; 95\% CI, 1.1 to 1.6 ) were more likely to have worked through their grief. In cases where health care staff offered parents counseling during the child's last month, the parents were more likely to have worked through their grief (fathers: RR 1.5; 95\% CI, 1.2 to 1.8; mothers; RR 1.2; 95\% CI, 1.1 to 1.4). Most parents eventually work through the grief associated with losing a child to cancer. In the long term, sharing the emotional burden with others facilitates the grieving process.

Improving Well-Being in Caregivers of Termi-
nally Ill Patients. Making the Case for Patient
Suffering as a Focus for Intervention Research

Hebert, R., Arnold, R., and Schulz, R.

Journal of Pain and Symptom Management, 34 (2007), 539-546

Family caregivers are integral to the care of patients with physical or mental impairments. Unfortunately, providing this care is often detrimental to the caregivers' health. As a result, in the last decade, there has been a proliferation of interventions designed to improve caregivers' well-being. Interventions for caregivers of persons at end of life, however, are relatively few in number and are often underdeveloped. They also are typically designed to help reduce the work of caregiving or to help caregivers cope with the physical and emotional demands of providing care. Although useful, these interventions generally ignore a primary stressor for family caregivers-a loved one's suffering. Patient suffering, whether physical, psychosocial, or spiritual, has a major impact on family caregivers. However, interventions that focus on the relief of patient suffering as a way to improve caregiver well-being have rarely been tested. It is our view that more research in this area could lead to new and more effective interventions for family caregivers of seriously or terminally ill patients. In support of our view, we define suffering and review the relationships between patient suffering and caregiver well-being. We then discuss a conceptual framework for intervention design. Finally, we conclude with a discussion of implications and future directions for intervention research. 